\title{
Random Response of Rotor System on the Filtered Gaussian White Noise Earthquake Ground Acceleration
}

\author{
Wang Zheng-Hao*, Xiong Xing-Rong, Meng Qing-Xin, Yang Xing-Tao and Xi Qing-Tai
}

School of Traffic and Mechanical Engineering, Shenyang Jianzhu University, Shenyang 110168, China

\begin{abstract}
With the pseudo-excitation method, the random response of a cantilever double-disc rotor system under the filtered Gaussian white noise earthquake ground accelerated random excitations, is analyzed by computer numerical simulation. The results show that the random response is relatively strong during an earthquake of the horizontal direction while the cantilever disk vibration is the strongest. The inherent frequency of the site soil has a great effect on the random response in the horizontal direction, while the system frequency characteristics have a great effect on the random response in the vertical direction. The rotor imbalance random excitation intensity does not change the peak frequency of the response spectrum. However, the system random vibration response is large when the excitation intensity is in a high level state. It has a great impact on the low and high frequency of non-cantilever disc, the low frequency of the cantilever disc, the high-frequency of non-cantilever bearing vertical vibration and the high-frequency of cantilever bearing the intensity around the high-frequency random response.
\end{abstract}

Keywords: Earthquake, filtered Gaussian white noise, pseudo excitation method, random response, rotor system.

\section{INTRODUCTION}

The development of high performance and increased efficiency rotating machines has prompted the researchers worldwide to review and research rotating machines in a new light [1-7]. In the seismic design, it is very important to study the vibration characteristics of the generator under the earthquake conditions. The random response analysis of complex rotor system is important to ensure that the system is operated safely and reliably. Due to the difficulties and complexities, there is limited research conducted in this area and the numerical calculations for the problems are also rare $[8,9]$. The method for the analysis of rotor system random response is the Monte Carlo method and the power spectrum algorithm. The researchers are often discouraged by the drawback of these methods which involves complex calculations and the expensive computation time. However, the pseudo-excitation method which is proposed by Professor Lin Jiahao is an effective method to analyze the linear random vibration system $[10,11]$. This method transforms the stationary random excitation into steady state harmonic excitation. It transforms the non-stationary random excitation into the transient uncertainty excitation. Moreover, this method has an advantage of being simple, efficient and accurate. With the pseudo-excitation method, the cantilever double-disc rotor system under the filtered Gaussian white noise earthquake ground acceleration random excitations is analyzed by computational numerical simulation. It provides the basis for rational selection and random control of the parameters of the rotor system.

*Address correspondence to this author at the School of Traffic and Mechanical Engineering, Shenyang Jianzhu University, Shenyang 110168, China; Tel: 13940126286; E-mail: wzh200864@126.com

\section{SYSTEM MECHANCIAL MODEL AND DIFFER- ENTIAL EQUATIONS OF MOTION}

Mechanical model of the rotor system is given in Fig. (1). We assume that casing is an elastic ring of linear radial contact stiffness. Stochastic differential equations of motion for the system under the effect of random excitation force are given in equation 1.

$M \ddot{u}+(C+G) \dot{u}+K u=F$

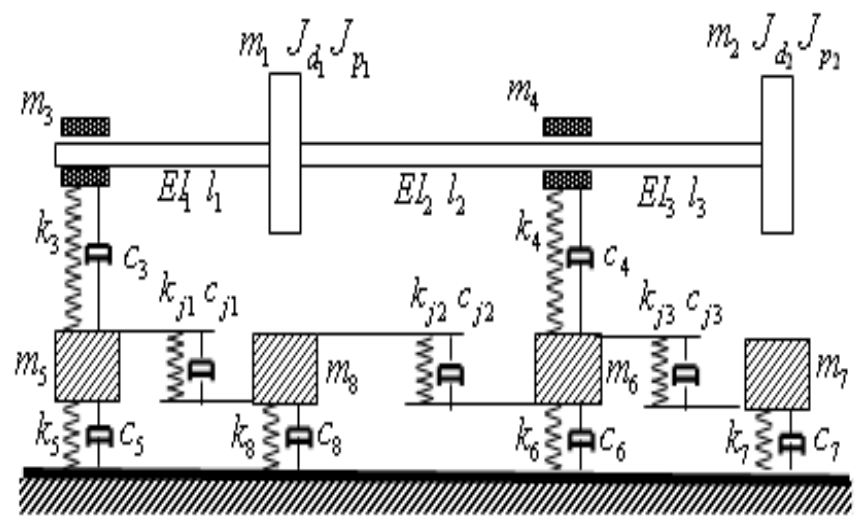

Fig. (1). Mechanical model of system.

The displacement array, random excitation force array, the mass matrix, the gyroscopic matrix, the damping matrix and the stiffness matrix are given, respectively.

$u=\left[u_{r}, u_{34}, u_{5678}\right]^{T}$

and $u_{r}=\left[x_{1}, \theta_{y_{1}}, x_{2}, \theta_{y_{2}}, y_{1}, \theta_{x_{1}}, y_{2}, \theta_{x_{2}}\right]^{T}$, 
$u_{34}=\left[x_{3}, y_{3}, x_{4}, y_{4}\right]^{T} \quad u_{5678}=\left[x_{5}, y_{5}, x_{6}, y_{6}, x_{7}, y_{7}, x_{8}, y_{8}\right]^{T}$

The random excitation force array is $F=F_{1}+F_{2}$, where $F_{l}$ includes gravity and the rotor imbalance random excitation force. $F_{2}$ is the ground random earthquake excitation. T $F_{1}$ can be expressed as

$F_{1}=\left[F_{1 x}, 0, F_{2 x}, 0, F_{1 y}, 0, F_{2 y}, 0, F_{34}, F_{56}, F_{78}\right]^{T}$

$F_{1 x}=m_{11} e_{1} \omega^{2} \cos \left(\omega t+\phi_{1}\right)-m_{1} g$

$F_{2 x}=m_{22} e_{2} \omega^{2} \cos \left(\omega t+\phi_{2}\right)-m_{2} g$

$F_{1 y}=m_{11} e_{1} \omega^{2} \sin \left(\omega t+\phi_{1}\right) F_{2 y}=m_{22} e_{2} \omega^{2} \sin \left(\omega t+\phi_{2}\right)$

$F_{34}=\left[-m_{3} g-m_{4} g\right]^{T} \quad F_{56}=\left[-m_{5} g-m_{6} g\right]^{T}$

$F_{78}=\left[-m_{7} g, 0,-m_{8} g, 0\right]^{T}$

where $\phi_{1}$ and $\phi_{2}$ are random variables of uniformly distributed $[0 \sim 2 \pi]$.

The $F_{2}$ can be expressed as

$F_{2}=-M E \ddot{Z}_{g}(t)$

where the $Z(\mathrm{t})$ is the ground acceleration random earthquake. We assume that $\mathrm{z}(\mathrm{t})$ is the filtered Gaussian white noise earthquake ground acceleration random excitations process, with the power spectral density given by [12]

$$
S_{z z}(\omega)=\frac{1+4\left(\frac{\xi_{g} \omega}{\omega_{g}}\right)^{2}}{\left(1-\frac{\omega^{2}}{\omega_{g}^{2}}\right)^{2}+4\left(\frac{\xi_{g} \omega}{\omega_{g}}\right)^{2}} \cdot \frac{1}{1+\frac{\omega^{2}}{\omega_{r}^{2}}} \cdot S_{o}
$$

where $\omega_{g}$, and $\xi_{g}$ are the inherent angular frequency of site soil and damping ratio, respectively. $\omega_{r}$ are the parameters of reaction bedrock spectral characteristics and $S_{o}$ is the bedrock acceleration power spectral density which is a spectral parameter of the reaction of ground motion intensity level. The stationary white noise earthquake excitation is given by $S_{z z}(\omega)=S_{o}$. For hard surface soil, the inherent angular frequency of the site and damping ratio are given by $\omega_{g}=5 \pi, \xi_{g}=0.63, \omega_{r}=8 \pi$. Fig. (2) gives an example of the filtered Gaussian white noise random excitations earthquake spectrum. In Fig. (2), the peak frequency is $11 \mathrm{rad} / \mathrm{s}$, the spectral peak is $1.438 S_{o}$.

Analysis shows that random response of rotor system is relatively strong during an earthquake of the horizontal direction, so inertial force instruction vector can be derived as

$$
\begin{aligned}
& E=\left[\begin{array}{llllllllllllllllllll}
1 & 0 & 1 & 0 & 0 & 0 & 0 & 0 & 1 & 0 & 1 & 0 & 1 & 0 & 1 & 0 & 1 & 0 & 1 & 0
\end{array}\right]^{T} \\
& M=\operatorname{diag}\left(M_{r}, M_{r}, M_{34}, M_{5678}\right)
\end{aligned}
$$

and $M_{r}=\operatorname{diag}\left(m_{1}, J_{d_{1}}, m_{2}, J_{d_{2}}\right)$,

$M_{34}=\operatorname{diag}\left(m_{3}, m_{3}, m_{4}, m_{4}\right)$,

$M_{5678}=\operatorname{diag}\left(m_{5}, m_{5}, m_{6}, m_{6}, m_{7}, m_{7}, m_{8}, m_{8}\right)$

$G=\left[\begin{array}{ll}G_{1} & 0 \\ 0 & 0\end{array}\right]$

and $G_{1}=\left[\begin{array}{lr}0 & -w J \\ w J & 0\end{array}\right] J=\operatorname{diag}\left(0, J_{P_{1}}, 0, J_{P_{2}}\right)$

$C=\left[\begin{array}{ccc}C_{r} & C_{12} & 0 \\ C_{12}^{T} & C_{123456} & 0 \\ 0 & 0 & C_{78}\end{array}\right]$

and $C_{r}=\operatorname{diag}\left(C_{e_{1}}+C_{c}, 0, C_{e_{2}}+C_{c}, 0, C_{e_{1}}+C_{c}, 0, C_{e_{2}}+C_{c}, 0\right)$

$C_{12}=\left[\begin{array}{ll}C_{121} & 0 \\ C_{122} & 0\end{array}\right], C_{121}$ and $C_{122}$ are cross-damping matrix.

$C_{78}=\operatorname{diag}\left(C_{7 x}+C_{j 3 x}, C_{7 y}+C_{j 3 y}, C_{8 x}+C_{j 1 x}+C_{j 2 x}, C_{8 y}+C_{j 1 y}+C_{j 2 y}\right)$

$C_{123456}$ is 8 rows and 8 columns symmetric matrix, the elements on main diagonal are

$\left(C_{3 x}, C_{3 y}, C_{4 x}, C_{4 y}, C_{3 x}+C_{5 x}+C_{j 1 x}+C_{j 3 y}, C_{3 y}+C_{5 y}+C_{j 1 y}\right.$,

$C_{4 x}+C_{6 x}+C_{j 2 x}+C_{j 3 x}, C_{4 y}+C_{6 y}+C_{j 2 y}+C_{j 3 y}$ )

$-C_{3 x}$ is the $5^{\text {th }}$ element of first row, . $-C_{3 y}$. is the $6^{\text {th }}$ element of second row, $-C_{4 x}$ is the $7^{\text {th }}$ element of third row, others are zero.

$K=\left[\begin{array}{ccccc}K_{r_{1}} & 0 & K_{1} & 0 & 0 \\ 0 & K_{r_{2}} & K_{2} & 0 & 0 \\ K_{1}^{T} & K_{2}^{T} & K_{3}-K_{3} & 0 \\ 0 & 0 & -K_{3}^{T} & K_{4} & 0 \\ 0 & 0 & 0 & 0 & K_{5}\end{array}\right]$

$k_{r_{1}}$ and $k_{r_{2}}$ are determined by rotor flexibility matrix, $k_{1}$ and $k_{2}$ are cross-stiffness matrix.

$$
\begin{aligned}
k_{3}= & \operatorname{diag}\left(k_{3 x}, k_{3 y}, k_{4 x}, k_{4 y}\right) \\
k_{4}= & \operatorname{diag}\left(k_{3 x}+k_{5 x}+k_{j 1 x}, k_{3 y}+k_{5 y}+k_{j 1 y},\right. \\
& \left.k_{4 x}+k_{6 x}+k_{j 2 x}+k_{j 3 x}, k_{4 y}+k_{6 y}+k_{j 2 y}+k_{j 3 y}\right) \\
k_{5}= & \operatorname{diag}\left(k_{7 x}+k_{j 3 x}, k_{7 y}+k_{j 3 y}, k_{8 x}+k_{j 1 x}+k_{j 2 x}, k_{8 y}+k_{j 1 y}+k_{j 2 y}\right)
\end{aligned}
$$

\section{ANALYSIS OF PSEUDO-EXCITATION OF RANDOM RESPOSE OF SYSTEM}

According to the basic principle of the pseudo-excitation method, the pseudo-excitation is created. Pseudo-vibration of system can be obtained by random differential equation of system. 
$M \frac{d^{2} \tilde{u}}{d t^{2}}+(C+G) \frac{d \tilde{u}}{d t}+K \tilde{u}=\tilde{F}$

where the pseudo-excitation can be expressed as

$\tilde{F}=\tilde{F}_{1}+\tilde{F}_{2}$

Solving virtual vibration differential equations, we can get virtual response

$\tilde{u}=\left[\tilde{u}_{r}, \tilde{u}_{34}, \tilde{u}_{5678}\right]^{T}$

and $\tilde{u}_{r}=\left[\tilde{x}_{1}, \tilde{\theta}_{y_{1}}, \tilde{x}_{2}, \tilde{\theta}_{y_{2}}, \tilde{y}_{1}, \tilde{\theta}_{x_{1}}, \tilde{y}_{2}, \tilde{\theta}_{x_{2}}\right]^{T}$

$\tilde{u}_{34}=\left[\tilde{x}_{3}, \tilde{y}_{3}, \tilde{x}_{4}, \tilde{y}_{4}\right]^{T}$

$\tilde{u}_{5678}=\left[\tilde{x}_{5}, \tilde{y}_{5}, \tilde{x}_{6}, \tilde{y}_{6}, \tilde{x}_{7}, \tilde{y}_{7}, \tilde{x}_{8}, \tilde{y}_{8}\right]^{T}$

The displacement power spectral matrix of random response is given by

$S_{v v}=\tilde{u}^{*} \tilde{u}^{T}$

where the superscript "*”" represents the complex conjugate numerical calculation.

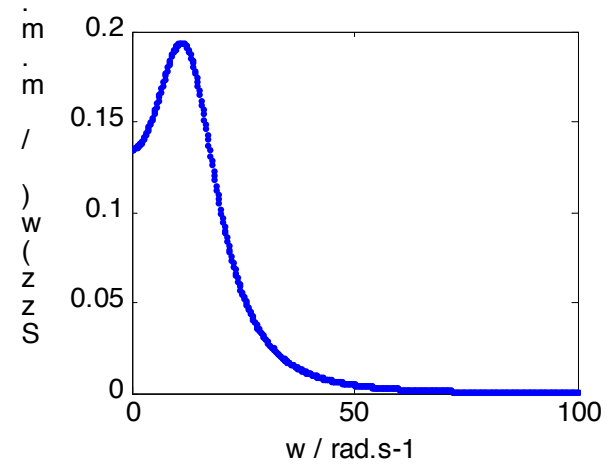

Fig. (2). Gaussian filtered white noise earthquake spectrum.

\section{NUMERICAL CALCULATION OF SYSTEM RANDOM RESOPNSE}

The standard values are inserted in numerical calculation of random response, such as, density of the material of shaft is $\rho=7.8 \times 10^{3} \mathrm{~kg} / \mathrm{m}^{3}$, the elastic modulus is $E=210 \times 10^{9} \mathrm{~N} / \mathrm{m}^{2}$, the three sections of shaft diameter are $d_{i}=30 \mathrm{~mm} \quad(i=1,2,3)$, the three section axis moment of inertia are $I_{i}=\pi d_{i}^{4} / 64 \quad(i=1,2,3)$, the three sections of shaft length are $l_{1}=210 \mathrm{~mm}, 、 l_{2}=330 \mathrm{~mm}$ and $l_{3}=240 \mathrm{~mm}$, the general mass is $m_{3}=3 \mathrm{~kg}$ and $m_{4}=3 \mathrm{~kg}$, the disc quality is $m_{i i}=24.3353 \mathrm{~kg} \quad(i=1,2)$, the disc equivalent mass is $m_{1}=25.9009 \mathrm{~kg}$ and $m_{2}=24.7251 \mathrm{~kg}$, respectively, the radius is $R_{i}=120 \mathrm{~mm} \quad(i=1,2)$, the thickness is $B_{i}=69 \mathrm{~mm}$ $(i=1,2)$, the polar moment of inertia is $J_{P i}=m_{i i} \cdot R_{i}^{2} / 2$ $(i=1,2)$, the moment of inertia about the diameter is $J_{d i}=J_{p i} / 2$, the casing quality is $m_{i}=2 m_{1} \quad(i=5,6,7,8)$, the roll damping is $C_{i x}=C_{i y}=0 \quad(i=3,4)$, the rolling bearing is the deep groove ball bearing, $\alpha=0.36$, the casing damping is $C_{i x}=C_{i y}=20 \mathrm{Ns} / \mathrm{m} \quad(i=5,6,7,8)$, the material internal damping is $C_{c}=20 \mathrm{Ns} / \mathrm{m}$, the outside damping is $C_{e i}=C_{e}=350 \mathrm{Ns} / \mathrm{m} \quad(i=1,2)$, the casing stiffness is $k_{i x}=k_{i y}=k_{j x}=10^{8} \mathrm{~N} / \mathrm{m} \quad(i=5,6,7,8)$, the rolling stiffness is $\quad k_{j i x}=k_{j i y}=k_{j}=10^{7} \mathrm{~N} / \mathrm{m} \quad(i=1,2,3) \quad$ and $\quad($ $\left.k_{i x}=k_{i y}=k=6 \times 10^{7} \mathrm{~N} / \mathrm{m}\right)(i=3,4)$, the stationary white noise earthquake excitation spectral density is $S_{O}=1.35 \times 10^{-1} \mathrm{~m}^{2} / \mathrm{s}^{3}$. For hard surface soil, the inherent angular frequency of site soil and damping ratio are $\omega_{g}=5 \pi$ and $\xi_{g}=0.63$, The parameters of bedrock spectral characteristics is $\omega_{r}=8 \pi$ and the speed is $n=8000 \mathrm{r} / \mathrm{min}$.

\subsection{Random Response of System Under the Filtered Gaussian White Noise Earthquake Spectrum}

Not considering rotor imbalance random excitation (the disc eccentricity is $e_{1}=e_{2}=e=0$ ), the system under power spectral density of random response of Gaussian filtered white noise earthquake spectrum excitation is shown in Fig. (3). The first circular frequency of a spectral peak $(11 \mathrm{rad} / \mathrm{s})$ is the peak frequency of filtered Gaussian white noise earthquake spectrum. It reflects the inherent frequency of site soil, the second circular frequency of a spectral peak ( $72 \mathrm{rad} / \mathrm{s}$ ) is the cantilever disk vertical critical angular velocity, it reflects system frequency characteristics. In Fig. (3), it is clear that the inherent frequency of the site soil has a great effect on the random response in the horizontal direction (y direction), but less impact in the vertical direction (x direction). The system frequency characteristic has a great effect on the random response in the vertical direction, but it has less impact in the horizontal direction. Due to the constraint of cantilever disk 2 , the response is relatively weakso the random vibration response of disk 2 is the strongest one. . Owingto the filtering effect of surface soil, the vibration energy of medium and high end seismic waves have been filtered out. The vibrational energy of random response is mainly concentrated in the low frequency band. The high frequency band of the vibrational energy is relatively small.

\subsection{Random Response of System Under Rotor Imbalance Random Excitation and Filtered Gaussian White Noise Earthquake Spectrum Excitation}

Power spectral density of random response about disc and bearing under rotor imbalance random excitation and filtered Gaussian white noise earthquake spectrum excitation is shown in Fig. (4). In Fig. (4), system has a certain amount of vibration energy in low, medium and high-end under rotor imbalance random excitation. When the eccentric moment is relatively small $(e<0.005 \mathrm{~mm})$, rotor imbalance random excitation has little effect on the random response. On the other hand, when the eccentric moment is relatively large $(e \geq 0.005 \mathrm{~mm})$, the effect cannot be ignored. Thus he rotor imbalance random excitation is stronger, random response is more intense, but it does not change the peak frequency of the response spectrum. The specific circumstances are as follows, 
(1) The rotor imbalance random excitation is stronger. The intensity of the low and high frequency of noncantilever disc 1 has improved. The intensity of the low frequency random response of cantilever disc 2 has improved but intensity of the high frequency random response has changed little.

(a)

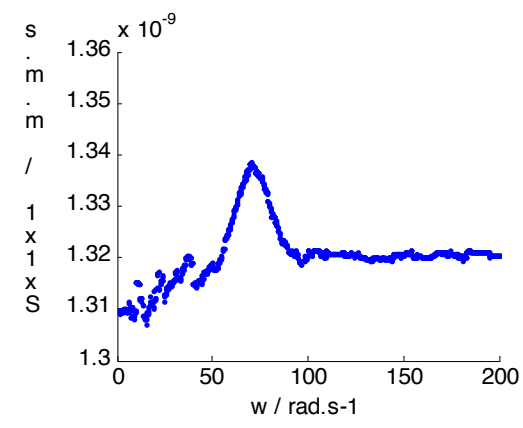

(b)

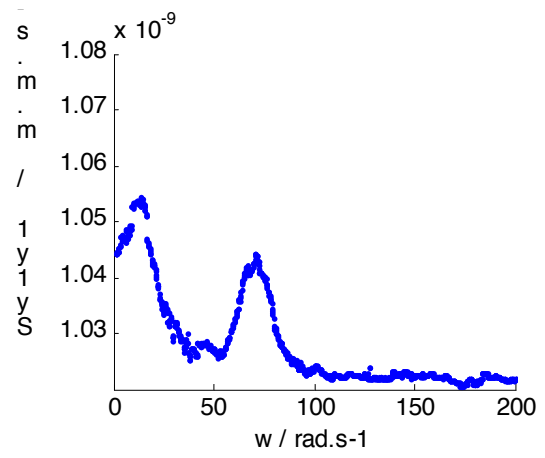

(c)

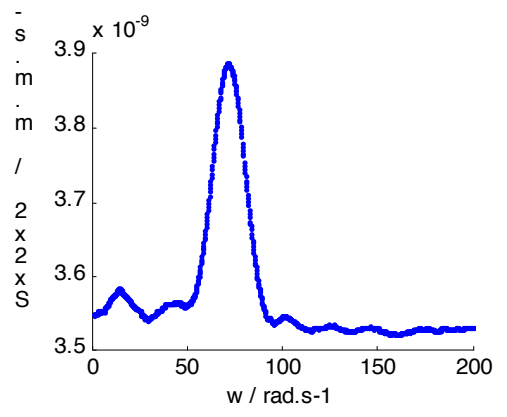

(d)

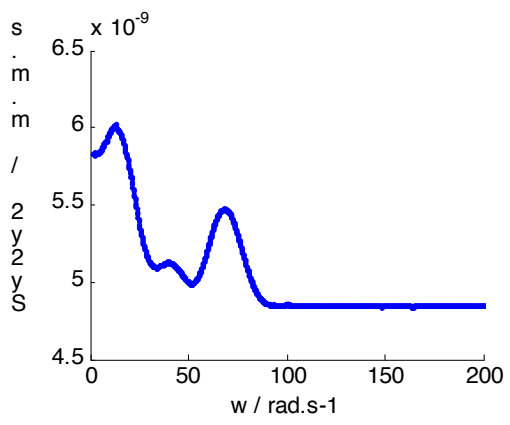

Fig. (3). random response of the system under Gaussian filtered white noise. (a)

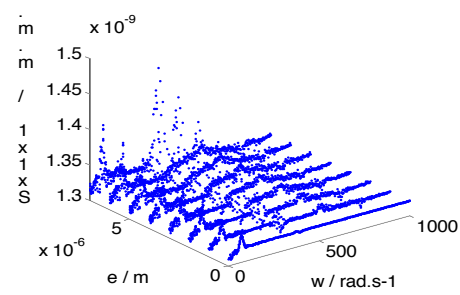

(b)

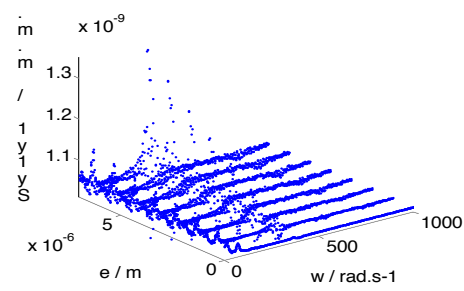

(c)

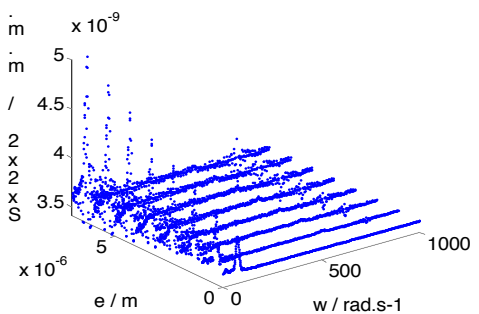

(d)

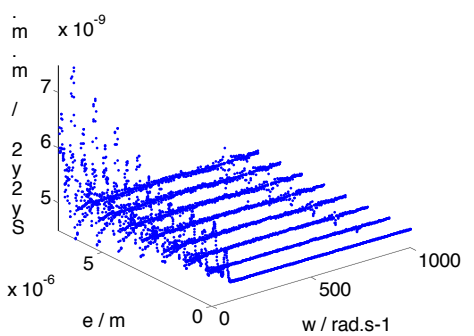

(e)

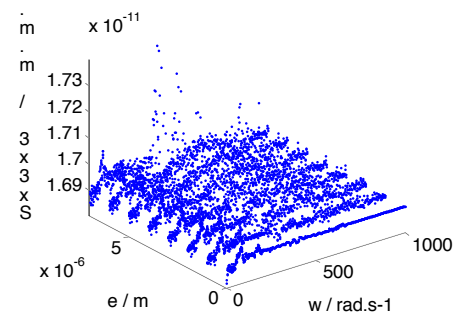

(f)

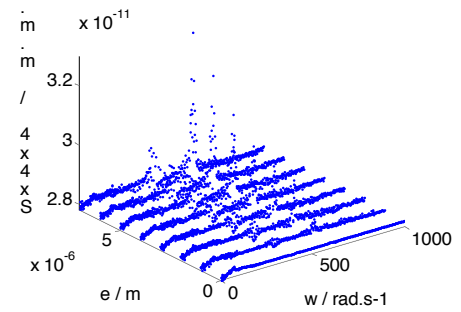

Fig. (4). Rotor imbalance random excitation effect on random response. 
(2) With the rotor imbalance random excitation enhanced for cantilever disc bearing 3 , the intensity of vertical high frequency random response increases rapidly. But the intensity of vertical low frequency random response exhibits little change. The intensity of the horizontal low frequency random response is improved, but the intensity of high frequency random response exhibits little change.

(3) The rotor imbalance random excitation is stronger and the high frequency random response of cantilever bearing 4 is more intense. The intensity of low frequency random response exhibits little change.

Integrating the above analysis the main conclusion we get is that

a. Under the rotor unbalance random excitation, the system has certain vibrational energy, irrespective of high, medium or low frequency range.

b. The rotor unbalance random excitation intensity doesn't change the peak frequency to make the strength of the random response increase. Especially when eccentric torque is equal to or more than 0.005 $\mathrm{Nm}(e \geq 0.005 \mathrm{~mm})$, the rotor unbalance random excitation on the influence of random response cannot be ignored.

c. The rotor unbalance random excitation has much influence on the high and low frequency of disk 1, the low frequency of disc 2, the vertical vibration high frequency of bearing 3 and the high frequency random response strength of bearing 4 .

\section{CONCLUSION}

It is appropriate that the method of pseudo-excitation is applied in the analysis of the rotor system in rotor imbalance random excitation and the filtered Gaussian white noise earthquake ground acceleration. Summary of the main conclusionobtained through numerical simulation is that:

As the cantilever disk is constrained relatively weak, the random vibration response is the most intense.

The inherent frequency of the site soil has a great effect on the horizontal direction of the random response, while the system frequency has a great effect on the vertical direction of the random response.

(3) The intensity of the rotor imbalance random excitation does not change the frequency peak of response spectrum. When the excitation intensity is high, the system random vibration response is large. It has a great impact on the low and high frequency of non-cantilever disc. The lower frequency of the cantilever disc, the higher the frequency of the non- cantilever bearing vertical vibration and the higherthe frequency of the cantilever bearing intensity around the high-frequency random response.

\section{CONFLICT OF INTEREST}

Financial contributions to the work being reported should be clearly acknowledged, as should any potential conflict of interest.

\section{ACKNOWLEDGEMENTS}

I would like to express my gratitude to all those who helped me during the writing of this thesis.

High tribute shall be paid to Ms. Wang Dan, whose profound knowledge of English triggers my love for this beautiful language and whose earnest attitude tells me how to learn English.

Finally, special thanks should go to my friends who have put considerable time and effort into their comments on the draft.

\section{REFERENCES}

[1] W. Bangchun, W. Xinhua, and D. Qian, "Theory and Experiment of Nonlinear Dynamics for Rotating Machinery with Faults", Science Press, pp. 2-4, 2004.

[2] S. Yanbe, "Rotor vibration due to collision with annular guard during passage through critical speed", ASME Journal of Vibration and Acoustics, vol. 120, pp. 544-550, 1998.

[3] Y. Huiqun, L. He, and W. Bangchun, "Study on stability and bifurcation behavior of the rotor with local rubbing", Proceedings of the First International Conference on the Integration of Dynamics, Monitoring and Control, 1999, pp.305-308.

[4] W. Bangchun, and Y. Hongliang, "Dynamics of rotor-bearing system with coupling faults of pedestal looseness and rub-impact", Proceedings of the $11^{\text {th }}$ World Congress on Theory of Machine and Mechanism, 2004, pp. 156-165.

[5] A. Sankaravelu, S.T. Noah, and C.P. Burger, "Bifurcation and chaos in ball bearings", Nonlinear and Stochastic Dynamics, vol. 192, no. 78, pp. 313-325, 1994.

[6] Y. Wang, "Prediction of periodic response of rotor dynamics stems with nonlinear supports", ASME Journal of Vibration and Acoustics, vol. 119, pp. 346-353, 1997.

[7] Z. Yimin, and W. Bangchun, "Reliability sensitivity for rotor-stator systems with rubbing", Journal of Sound and Vibration, vol. 259, no. 5, pp. 1095-1107, 2003.

[8] Z. Yan, L. Jiahao, and C. Jianhua, "Variation analysis of shaft systems subjected to stationary random seismic excitations", Engineering Mechanics, vol. 19, no. 2, pp. 26-31, 2002.

[9] Z. Yan, L. Jiahao, and C. Jianhua, "Seismic analysis of rotor system under stationary nonstationary random earthquake excitations", Journal of Computational Mechanics, vol. 19, no. 1, pp. 8-11, 2002

[10] L. Jiahao, and Z. Wenshou, "Some noteson FEM and structural random response analysis", Journal of Computational Mechanics, vol. 15 , no. 2 , pp. $217-223,1998$.

[11] L. Jiahao, Z. Wenshou, and L. Jianjun, "Structural response to arbitrarily coherent stationary random excitations", Computers \& Structures, vol. 50, no. 5, pp. 629-634, 1994.

[12] O. Jinping, and W.Guangyuan, "Random Vibration of Structures", Higher Education Press, vol. 124, 1998.

(C) Zheng-Hao et al.; Licensee Bentham Open

This is an open access article licensed under the terms of the Creative Commons Attribution Non-Commercial License (http://creativecommons.org/licenses/ by-nc/4.0/) which permits unrestricted, non-commercial use, distribution and reproduction in any medium, provided the work is properly cited. 